\title{
Centralizer of the elementary subgroup of an isotropic reductive group
}

\author{
E. Kulikova*, A. Stavrova ${ }^{\dagger}$
}

October 31, 2018

\section{Introduction}

Let $R$ be a commutative ring with 1 , and let $G$ be an isotropic reductive algebraic group over $R$. In [5] Victor Petrov and the second author introduced a notion of an elementary subgroup $E(R)$ of the group of points $G(R)$.

More precisely, assume that $G$ is isotropic in the following strong sense: it possesses a parabolic subgroup that intersects properly any semisimple normal subgroup of $G$. Such a parabolic subgroup $P$ is called strictly proper. Denote by $E_{P}(R)$ the subgroup of $G(R)$ generated by the $R$-points of the unipotent radicals of $P$ and of an opposite parabolic subgroup $P^{-}$. The main theorem of [5] states that $E_{P}(R)$ does not depend on the choice of $P$, as soon as for any maximal ideal $M$ of $R$ all irreducible components of the relative root system of $G_{R_{M}}$ (see [2, Exp. XXVI, §7] for the definition) are of rank $\geq 2$. Under this assumption, we call $E_{P}(R)$ the elementary subgroup of $G(R)$ and denote it simply by $E(R)$. In particular, $E(R)$ is normal in $G(R)$. This definition of $E(R)$ generalizes the well-known definition of an elementary subgroup of a Chevalley group (or, more generally, of a split reductive group), as well as several other definitions of an elementary subgroup of isotropic classical groups and simple groups over fields. The group $E(R)$ is also perfect under natural assumptions on $R$ [3. Here we continue this theme by proving that the centralizer of $E(R)$ in $G(R)$ coincides with the group of $R$-points of the group scheme center $\operatorname{Cent}(G)$ (see [2, Exp. I 2.3] for the definition). Consequently, both these subgroups also coincide with the abstract group center of $G(R)$. Our result extends the respective theorem of E. Abe and J. Hurly for Chevalley groups [1]; see also [7, Lemma 2] for a slighly more general statement.

Theorem 1. Let $G$ be an isotropic reductive algebraic group over a commutative ring $R$ having a strictly proper parabolic subgroup $P$. Assume that for any maximal ideal $M$ of $R$ all irreducible components of the relative root system of $G_{R_{M}}$ are of rank $\geq 2$. Then $\mathrm{C}_{G(R)}(E(R))=\operatorname{Cent}(G)(R)=\mathrm{C}(G(R))$.

Observe that the condition of the theorem ensures that the elementary subgroup $E(R)$ of $G(R)$ is correctly defined. We refer to [3] for its definition and basic properties, as well as for the preliminaries on relative root subschemes.

Remark. One may ask if the statement holds for $E_{P}(R)$ instead of $E(R)$, if we do not assume that the local relative rank is at least 2. This seems to hold always except for several natural exceptions, similar to the exception for $\mathrm{PGL}_{2}$ described in [1. We plan to address this case in the near future.

\footnotetext{
* The author is supported by RFBR 08-01-00756.

$\dagger$ The author is supported by RFBR 09-01-00878 and RFBR 09-01-90304.
} 


\section{Preliminary lemmas}

We refer to [3] and [5] for the preliminaries and notation.

We include the following obvious lemma for the sake of completeness.

Lemma 1. Let $X=\operatorname{Spec} A$ be an affine scheme over $Y=\operatorname{Spec} R$, and let $Z$ be a closed subscheme of $X$. Take $g \in X(R)$. Then $g \in Z(R)$ if and only if $g \in Z\left(R_{M}\right)$ for any maximal ideal $M$ of $R$.

Proof. For any $R$-module $V$, the natural map $V \rightarrow \prod V \otimes R_{M}$, where the product runs over all maximal ideals $M$ of $R$, is injective (e.g. [8, p. 104, Lemma]). Since $g \in Z(R)$ is equivalent to an inclusion between the respective ideals of $A$ which are $R$-modules, the Lemma holds.

Lemma 2. Let $R$ be any commutative ring, $G$ an isotropic reductive group over $R, P$ a strictly proper parabolic subgroup of $G$. Take any maximal ideal $M$ of $R$ and any strictly proper parabolic subgroup $P^{\prime}$ of $G_{R_{M}}$ contained in $P_{R_{M}}$. Then for any $A \in \Phi_{P^{\prime}}$ there is a system of generators $e_{A i}, 1 \leq i \leq n_{A}$, of the $R_{M}$-module $V_{A}$ such that for all $g$ in the image of $\operatorname{Cent}_{G(R)}\left(E_{P}(R)\right)$ in $G\left(R_{M}\right)$, one has $\left[g, X_{A}\left(e_{A i}\right)\right]=1,1 \leq i \leq n_{A}$.

Proof. We assume from the very beginning that we have passed to a member of the disjoint union

$$
\operatorname{Spec}(R)=\coprod_{i=1}^{m} \operatorname{Spec}\left(R_{i}\right),
$$

so that the parabolic subgroup $P$ is also provided with a relative root system $\Phi_{P}$ and corresponding relative root subschemes. Since for any $B \in \Phi_{P}$ elements of $V_{B}$ generate $V_{B} \otimes_{R} R_{M}$ as an $R_{m}$-module, the claim of the lemma holds if $P^{\prime}=P_{R_{M}}$.

By [5. Lemma 12], for any two strictly proper parabolic subgroups $Q \leq Q^{\prime}$ of a reductive group scheme, one can find such $k>0$ depending only on $\operatorname{rank} \Phi_{Q}$, that for any relative root $A \in \Phi_{Q}$ and any $v \in V_{A}$ there exist relative roots $B_{i}, C_{i j} \in \Phi_{Q^{\prime}}$, elements $v_{i} \in V_{B_{i}}$, $u_{i j} \in V_{C_{i j}}$, and integers $k_{i}, n_{i}, l_{i j}>0\left(1 \leq i \leq m, 1 \leq j \leq m_{j}\right)$, which satisfy the equality

$$
X_{A}\left(\xi \eta^{k} v\right)=\prod_{i=1}^{m} X_{B_{i}}\left(\xi^{k_{i}} \eta^{n_{i}} v_{i}\right)^{m_{i}} X_{C_{i j}}\left(\eta^{l_{i j}} u_{i j}\right)
$$

where $\xi, \eta$ are free variables. Taking $Q=P^{\prime}, Q^{\prime}=P_{R_{M}}, \xi=1$, for any element $v_{i}$ of a generating system of the $R_{m}$-module $V_{A}$ we get a decomposition

$$
X_{A}\left(\eta^{k} v\right)=\prod_{i=1}^{m} X_{B_{i}}\left(\eta^{n_{i}} v_{i}\right)
$$

for some $B_{i} \in \Phi_{P}$ and $v_{i} \in V_{B_{i}} \otimes R_{M}, n_{i}>0$. Clearly, for any $v_{i}$ there is an element $s_{i} \in R \backslash M$ such that $s_{i} v_{i}$ belongs to $V_{B_{i}}$ (strictly speaking, to the image of $V_{B_{i}}$ in $V_{B_{i}} \otimes R_{M}$ under the localisation homomorphism; here and below we allow ourselves this freedom of speech). Set $\eta=s_{1} \ldots s_{m}$. Then $X_{A}\left(\eta^{k} v\right) \in E_{P}(R)$, and hence $\left[g, X_{A}\left(\eta^{k} v\right)\right]=1$ for any $g \in \operatorname{Cent}_{G(R)}\left(E_{P}(R)\right)$. Thus, multiplying the elements of a generating system of $V_{A}$ by certain invertible elements of $R_{M}$, we obtain a new generating system of $V_{A}$, which is centralized by $\operatorname{Cent}_{G(R)}\left(E_{P}(R)\right)$.

Lemma 3. Let $R$ be a local ring (in particular, $R$ can be a field) with the maximal ideal $M$, and let $G$ be a split reductive group over $R$. Let $P$ be a parabolic subroup of $G$ such that $\operatorname{rank} \Phi_{P} \geq 2$. Assume that $g \in G(R)$ is such that for any $A \in \Phi_{P}$ there is a system of generators $e_{A i}, 1 \leq i \leq n_{A}$, of $V_{A}$ such that $\left[g, X_{A}\left(e_{A i}\right)\right]=1$ for all $i$. Then $g \in$ $U_{P}(M) L(R) U_{P^{-}}(M)$, where $U_{P^{ \pm}}(M)=\left\langle X_{A}\left(M V_{A}\right), A \in \Phi^{ \pm}\right\rangle$. 
Proof. First let $R$ be a field. We need to show that $g \in L(R)$. We can assume that $R$ is algebraically closed without loss of generality. Let $B^{ \pm}$be opposite Borel subgroups of $G$ contained in $P^{ \pm}, U^{ \pm}$be their unipotent radicals, and $T$ their common maximal torus. Bruhat decomposition implies that $g=u h w v$, where $u \in U^{+}(R), h \in T(R), w$ is a representative of the Weyl group, $v \in U_{w}^{+}(R)=\left\{x \in U^{+}(R) \mid w(x) \in U^{-}(R)\right\}$, and this decomposition is unique. We have $w \in L(R)$ if and only if $w$ is a product of elementary reflections $w_{\alpha_{i}}$ for some simple roots $\alpha_{i}$ belonging to the root system of $L$.

Assume first that $w \notin L$. Then there is a simple root $\alpha$ not belonging to the root system of $L$ such that $w(\alpha)<0$. Consider $A=\pi(\alpha)$. Let $e_{A} \in V_{A}$ be a vector from the generating set existing by the hypothesis of the Lemma such that $x_{\alpha}(\xi), \xi \neq 0$, occurs in the canonic decomposition of $x=X_{A}\left(e_{A}\right)$ into a product of elementary root unipotents from $U^{+}$. Since $[g, x]=1$, we have $x(u h w v)=(u h w v) x$. The rightmost factor in the Bruhat decomposition of $x(u h w v)=(x u) h w v$ equals $v$. However, since $\alpha$ is a positive root of minimal height, it is clear that the rightmost factor in the Bruhat decomposition of $(u h v) x$ contains $x_{\alpha}(\eta+\xi)$ in its canonic decomposition, if $v$ contains $x_{\alpha}(\eta)$. Therefore, this rightmost factor is distinct from $v$, a contradiction.

Therefore, $w \in L(R)$. Then for any $x \in U_{P}(R)$ we have $w x w^{-1} \in U_{P}(R)$, hence by the definition of the Bruhat decomposition $v \in L(R) \cap U^{+}(R)$. This means that $g=$ uhwv $\in U^{+}(R) L(R)=U_{P}(R)\left(U^{+}(R) \cap L(R)\right) L(R)=U_{P}(R) L(R)=P(R)$. Since symmetric reasoning implies that $g \in P^{-}(R)$, we have $g \in P(R) \cap P^{-}(R)=L(R)$.

Now let $R$ be any local ring. Recall that $\Omega_{P}=U_{P} L U_{P^{-}} \cong U_{P} \times L \times U_{P^{-}}$is a principal open subscheme of $G$ (e.g. [4, p. 9]). Therefore, if the image of $g \in G(R)$ under the natural homomorphism $G(R) \rightarrow G(R / M)$ is in $\Omega_{P}(R / M)$, then $g \in \Omega_{P}(R)$. Since by the above the image of $g$ is in $L(R / M)$, and $\operatorname{ker}\left(U_{P^{ \pm}}(R) \rightarrow U_{P^{ \pm}}(R / M)\right)=U_{P^{ \pm}}(M)$, we have $g \in U_{P}(M) L(R) U_{P^{-}}(M)$.

Lemma 4. Let $G$ be an isotropic reductive group over a local ring $R, M$ the maximal ideal of $R, P$ a parabolic subgroup of $G, P^{-}$an opposite parabolic subgroup. For any $u \in U_{P^{-}}(M)$, $v \in U_{P}(R)$ there exist $u^{\prime} \in U_{P^{-}}(M), v^{\prime} \in U_{P}(R)$, and $b \in L(R)$ such that $u v=v^{\prime} b u^{\prime}$.

Proof. The image of $x=u v$ under $p: G(R) \rightarrow G(R / M)$ equals $p(v)$, and thus belongs to $\Omega_{P}(R / M)$, where $\Omega_{P}=U_{P} L U_{P^{-}}$. Since $\Omega_{P}$ is a principal open subscheme of $G$, this implies that $x \in \Omega_{P}(R)$, that is, $x=v^{\prime} b u^{\prime}$. Since $p\left(u^{\prime}\right)=1$, we have $u^{\prime} \in U_{P^{-}}(M)$.

Lemma 5. Let $G$ be a reductive group over a commutative ring $R, P$ a parabolic subgroup of $G, A, B \in \Phi_{P}$ two non-proportional relative roots such that $A+B \in \Phi_{P}$. Assume that $A-B \notin \Phi_{P}$, or $A, B$ belong to the image of a simply laced irreducible component of the absolute root system of $G$. Take $0 \neq u \in V_{B}$. Any generating system $e_{1}, \ldots, e_{n}$ of the $R$-module $V_{A}$ contains an element $e_{i}$ such that $N_{A B 11}\left(e_{i}, u\right) \neq 0$.

Proof. Assume that $N_{A B 11}\left(e_{i}, u\right)=0$ for all $1 \leq i \leq n$. Consider an affine fpqc-covering $\amalg \operatorname{Spec} S_{\tau} \rightarrow \operatorname{Spec} R$ that splits $G$. There is a member $S_{\tau}=S$ of this covering such that the image of $X_{B}(u)$ under $G(R) \rightarrow G(S)$ is non-trivial. Write

$$
X_{B}(u)=\prod_{\pi(\beta)=B} x_{\beta}\left(a_{\beta}\right) \cdot \prod_{i \geq 2} \prod_{\pi(\beta)=i B} x_{\beta}\left(c_{\beta}\right),
$$

where $\pi: \Phi \rightarrow \Phi_{P}$ is the canonical projection of the absolute root system of $G$ onto the relative one, $x_{\beta}$ are root subgroups of the split group $G_{S}$, and $a_{\beta} \in S$. Since $X_{B}(u) \neq 0$, the definition of $X_{B}$ implies that there exists $a_{\beta} \neq 0$. Let $\beta_{0} \in \pi^{-1}(B)$ be the root of minimal height with this property. By [5, Lemma 4] there exists a root $\alpha \in \pi^{-1}(A)$ such that $\alpha+\beta_{0} \in \Phi$. Let $v \in V_{A} \otimes_{R} S$ be such that $X_{A}(v)=x_{\alpha}(1) \prod_{i \geq 2} \prod_{\pi(\gamma)=i A} x_{\gamma}\left(d_{\gamma}\right)$, for some $d_{\gamma} \in S$. Then the (usual) Chevalley commutator formula implies that $\left[X_{A}(v), X_{B}(u)\right]$ 
contains in its decomposition a factor $x_{\alpha+\beta}\left(\lambda a_{\beta_{0}}\right)$, where $\lambda \in\{ \pm 1, \pm 2, \pm 3\}$. However, since either $\alpha, \beta$ belong to a simply laced irreducible component of $\Phi$, or $A-B \notin \Phi_{P}$, we have $\lambda= \pm 1$. Then $N_{A B 11}(v, u) \neq 0$, a contradiction.

Recall [5] that any relative root $A \in \Phi_{J, \Gamma}$ can be represented as a (unique) linear combination of simple relative roots. The level $\operatorname{lev}(A)$ of a relative root $A$ is the sum of coefficients in this decomposition.

Lemma 6. Let $R$ be a local ring with the maximal ideal $M$, and let $G$ be a reductive group over $R$. Let $P$ be a parabolic subgroup of $G$ such that $\operatorname{rank} \Phi_{P} \geq 2$, and the type of $P$ occurs as the type of a minimal parabolic subgroup of some reductive group over a local ring (not necessarily over $R$ ). Assume that $g \in G(R)$ is such that for any $A \in \Phi_{P}$ there is a system of generators $e_{A i}, 1 \leq i \leq n_{A}$, of $V_{A}$ such that $\left[g, X_{A}\left(e_{A i}\right)\right]=1$ for all $i$. If $g \in U_{P}(M) L(R) U_{P^{-}}(M)$, then $g \in L(R)$.

Proof. Write $g=x h y$, where $x \in U_{P}(M), h \in L(R), y \in U_{P^{-}}(M)$. We have $\prod_{A \in \Phi_{P}^{+}} X_{A}\left(u_{A}\right)$, $y=\prod_{A \in \Phi_{P}^{-}} X_{A}\left(u_{A}\right)$, where the product is taken in any fixed order.

Let $A \in \Phi_{P}$ be such that $u_{A} \neq 0$, and $|\operatorname{lev}(A)|$ is minimal among the levels of relative roots with this property. We are going to deduce a contradiction, thus showing that $A$ cannot occur in the decomposition of $g$.

Assume that $A \in \Phi_{P}^{+}$; the other case is treated symmetrically. Since the type of $P$ coincides with the type of a minimal parabolic subgroup, $\Phi_{P}$ is isomorphic to a root system as a set with two partially defined operations-addition and multiplication by integers. Then the standard properties of a root system imply that one can find a simple root or a minus simple root $B \in \Phi_{P}$, non-proportional to $A$, such that $A+B \in \Phi_{P}$. Moreover, if the irreducible component of $\Phi_{P}$ containing $A$ is not of type $G_{2}$, we can, and we will, choose $B$ so that $A-B \notin \Phi_{P}$. If it is of type $G_{2}$, this may be impossible; then we stipulate that we take $B$ positive. The classification of Tits indices over local rings [6] also implies that in this case the respective irreducible component of the absolute root system of $G$ is either simply laced or itself of type $G_{2}$. Assume for now that the latter does not take place; we will treat this exceptional case in the very end of this proof. Then by Lemma 5 one can find an element $e$ of a generating system of $V_{B}$ centralized by $g$ such that $N_{A B 11}\left(u_{A}, e\right) \neq 0$.

We have $1=\left[X_{B}(e), g\right]=\left[X_{B}(e), x\right]\left(x\left[X_{B}(e), h y\right] x^{-1}\right)$. This is equivalent to

$$
1=\left(x^{-1}\left[X_{B}(e), x\right] x\right)\left[X_{B}(e), h y\right]=\left[x^{-1}, X_{B}(e)\right]\left[X_{B}(e), h y\right] .
$$

By [5, Th. 2] we can write

$$
x^{-1}=X_{A}\left(-u_{A}\right) \prod_{\substack{C \in \Phi_{P}^{+}, C \neq A, \operatorname{lev}(C) \geq \operatorname{lev}(A)}} X_{C}\left(v_{C}\right)=X_{A}\left(-u_{A}\right) \cdot x_{1},
$$

and thus

$$
\begin{aligned}
& {\left[x^{-1}, X_{B}(e)\right]=\left[X_{A}\left(-u_{A}\right) x_{1}, X_{B}(e)\right]} \\
& =\left[X_{A}\left(-u_{A}\right),\left[x_{1}, X_{B}(e)\right]\right] \cdot\left[x_{1}, X_{B}(e)\right] \cdot\left[X_{A}\left(-u_{A}\right), X_{B}(e)\right] .
\end{aligned}
$$

Case 1: $B$ is positive, that is, $B$ is a simple root. We study the factor $\left[X_{B}(e), h y\right]$ of (11). Write $\left[X_{B}(e), h y\right]=X_{B}(e) h\left(y X_{B}(e)^{-1} y^{-1}\right) h^{-1}$, and

$$
y=\prod_{C \in \Phi_{P}^{-}, C \nmid B} X_{C}\left(v_{c}\right) \cdot \prod_{i>0} X_{-i B}\left(v_{-i B}\right)=y_{1} y_{2} .
$$

Using Lemma4we obtain $y X_{B}(e)^{-1}=y_{1}\left(y_{2} \cdot X_{B}(e)^{-1}\right)=y_{1} \cdot \prod_{i>0} X_{i B}\left(w_{i B}\right) \cdot b \cdot \prod_{i>0} X_{-i B}\left(w_{i B}\right)$, where $b \in L(R)$. Since relative roots proportional to $B$ does not occur in the decompo- 
sition of $y_{1}$, and $B$ is a simple root, the generalized Chevalley commutator formula implies that $y_{1} \cdot \prod_{i>0} X_{i B}\left(w_{i B}\right)=\left(\prod_{i>0} X_{i B}\left(w_{i B}\right)\right) y_{3}$, where $y_{3} \in U_{P^{-}}(R)$. Hence $y X_{B}(e)^{-1} \in$ $\left(\prod_{i>0} X_{i B}\left(w_{i B}\right)\right) P^{-}(R)$, and also

$$
\left[X_{B}(e), h y\right] \in X_{B}(e) h\left(\prod_{i>0} X_{i B}\left(w_{i B}\right)\right) h^{-1} P^{-}(R)=\left(\prod_{i>0} X_{i B}\left(z_{i B}\right)\right) P^{-}(R) .
$$

Now we consider the first factor $\left[x^{-1}, X_{B}(e)\right]$ of the right side of (11). The generalized Chevalley commutator formula, applied to (2), says that

$$
\left[x^{-1}, X_{B}(e)\right]=\prod_{D \in \Phi_{P}^{+}} X_{D}\left(w_{D}\right) .
$$

Moreover, $D=A+B$ is a root of minimal height in the decomposition (2) satisfying $w_{D} \neq 0$; in fact, $w_{A+B}=N_{A B 11}\left(-u_{A}, e\right)$. Hence, the whole product

$$
\left[x^{-1}, X_{B}(e)\right] \cdot\left[X_{B}(e), h y\right] \in X_{A+B}\left(N_{A B 11}\left(-u_{A}, e\right)\right) \cdot\left(\prod_{i>0} X_{i B}\left(z_{i B}\right)\right) \cdot \prod_{C \in \Phi_{P}^{+},} X_{C}\left(t_{C}\right) \cdot P^{-}(R)
$$

does not equal 1 , a contradiction.

Case 2: $B$ is negative, that is $B^{\prime}=-B$ is a simple root. In this case the generalized Chevalley commutator formula immediately implies $\left[X_{B}(e), h y\right] \in P^{-}(R)$. We study (2). Note that the decomposition of $x_{1}$ does not contain $X_{B^{\prime}}\left(v_{B^{\prime}}\right)$, and, if $2 B^{\prime} \in \Phi_{P}$, also does not contain $X_{2 B^{\prime}}\left(v_{2 B^{\prime}}\right)$. Indeed, in the first case we would have $\operatorname{lev}(A)=1$, hence $A$ is a simple relative root, hence $A+B=A-B^{\prime}$ is not a relative root. In the second case we would have $\operatorname{lev}(A)=2$, and, since $A+B \in \Phi_{P}, A=A^{\prime}+B^{\prime}$ for a simple relative root $A^{\prime}$. Since in this case we are in the irreducible component of $\Phi_{P}$ of type $B C_{n}$, and $B^{\prime}$ is an extra-short simple root, we also have $A^{\prime}+2 B^{\prime}=A-B \in \Phi_{P}$. But then by our algorithm we would have taken $\left(-A^{\prime}\right)$ instead of $B$, since $A-\left(-A^{\prime}\right)=2 A^{\prime}+B^{\prime} \notin \Phi_{P}$.

The above, together with the fact that $B^{\prime}=-B$ is a simple root, and the generalized Chevalley commutator formula, implies that $\left[x_{1}, X_{B}(e)\right]=\prod_{D \in \Phi_{P}^{+}} X_{D}\left(w_{D}\right)$. Moreover, if $w_{D} \neq 0$, then $D \neq A+B$, since $A-B$ is not a relative root by our assumptions, and obviously $D$ is not proportional to $B$. Further, we see that for any relative root $D$, occuring in the decomposition of $\left[X_{A}\left(-u_{A}\right),\left[x_{1}, X_{B}(e)\right]\right]$ or $\left[X_{A}\left(-u_{A}\right), X_{B}(e)\right]$, the coefficient near any simple root $A_{0} \neq B^{\prime}$ in the decomposition of $D$ is greater or equal to that in the decomposition of $A$. Summing up, the only factor of the form $X_{A-B}(u)$ in the decompositions of the expressions $\left[X_{A}\left(-u_{A}\right),\left[x_{1}, X_{B}(e)\right]\right],\left[x_{1}, X_{B}(e)\right],\left[X_{A}\left(-u_{A}\right), X_{B}(e)\right]$ is the factor $X_{A-B}\left(N_{A B 11}\left(-u_{A}, e\right)\right)$ in the third one, and no commutator of the factors can give a new factor of the form $X_{A-B}(u)$ with $u \neq 0$. Hence, $\left[x^{-1}, X_{B}(e)\right]$ contains $X_{A-B}\left(N_{A B 11}\left(-u_{A}, e\right)\right) \neq 1$ in its decomposition, and

$$
\left[x^{-1}, X_{B}(e)\right]\left[X_{B}(e), h y\right] \in X_{A-B}\left(N_{A B 11}\left(-u_{A}, e\right)\right) \cdot \prod_{\substack{F \in \Phi_{P}^{+}, F \neq A-B}} X_{F}\left(t_{F}\right) \cdot P^{-}(R)
$$

cannot equal 1 , a contradiction.

Case $G_{2}$. We are left with the case when $\Phi_{P}$ is of type $G_{2}$, and moreover the relevant component of the absolute root system of $G$ is also of type $G_{2}$. Then we can assume without loss of generality that all components of the absolute root system are of type $G_{2}$, and consequently $G$ is quasi-split. There exists a canonical étale extension $R^{\prime}$ of $R$ such 
that $G$ is a Weil restriction of a split group $G^{\prime}$ of type $G_{2}$ over $R^{\prime}$, see 2, Exp. XXIV Prop. 5.9]. Then $G_{R^{\prime}}$ is a direct product of $k$ split groups $G_{i}$ of type $G_{2}$. To show that $g \in L(R)$, it is enough to show that the image $g^{\prime}$ of $g$ in $G\left(R^{\prime}\right)$ is in $L\left(R^{\prime}\right)$. We know that $P_{R^{\prime}}$ is a Borel subgroup of $G_{R^{\prime}}$, and, since $\Phi_{P}$ has no multiple roots, for any $A \in \Phi_{P}$ we can identify the root subscheme $X_{A}\left(V_{A} \otimes R^{\prime}\right)$ with the direct product of $k$ elementary root subgroups $x_{\alpha}\left(R^{\prime}\right)$ of the groups $G_{i}$. Considering the relevant projections of $g$ and the generating systems of $V_{A}$, we are reduced to proving the following: if a point $h \in H(S)$ of a split reductive group $H$ of type $G_{2}$ centralizes $x_{\alpha}\left(u_{\alpha}\right)$ for some $u_{\alpha} \in S^{\times}$, for any root $\alpha \in \Psi$, where $\Psi$ is the root system of $H$, then $h$ belongs to the corresponding split maximal torus. By Lemmas 1 and 3 we can also assume that the ring $S$ is local with the maximal ideal $N$, and $h=\prod_{\alpha \in \Psi^{+}} x_{\alpha}\left(a_{\alpha}\right) \cdot h \cdot \prod_{\alpha \in \Psi^{-}} x_{\alpha}\left(a_{\alpha}\right)$, where all $a_{\alpha} \in N$. Then the proof goes exactly as in [1, Prop. 3], substituting the elements $x_{\beta}(1)$ and $w_{\beta}(1)$ by $x_{\beta}\left(u_{\beta}\right)$ and $w_{\beta}\left(u_{\beta}\right)=x_{\beta}\left(u_{\beta}\right) x_{-\beta}\left(-u_{\beta}^{-1}\right) x_{\beta}\left(u_{\beta}\right)$.

Lemma 7. Let $G$ be an isotropic reductive algebraic group over a commutative ring $R, P$ a parabolic subgroup of $G, L$ a Levi subgroup of $P$. Assume that $g \in G(R)$ is such that for any $A \in \Phi_{P}$ there is a system of generators $e_{A i}, 1 \leq i \leq n_{A}$, of $V_{A}$ such that $\left[g, X_{A}\left(e_{A i}\right)\right]=1$ for all $i$. If $g \in L(R)$, then $\left[g, E_{P}(R)\right]=1$.

Proof. We show that $\left[g, X_{A}\left(V_{A}\right)\right]=0$ for any $A \in \Phi_{P}^{+}$by descending induction on the hight of $A$; the case $A \in \Phi_{P}^{-}$is symmetric. By [5, Th. 2] for any $g \in L(S)$ and any $A \in \Phi_{P}$ there exists a set of homogeneous polynomial maps $\varphi_{g, A}^{i}: V_{A} \rightarrow V_{i A}, i \geq 1$, such that for any $v \in V_{A}$ one has

$$
g X_{A}(v) g^{-1}=\prod_{i \geq 1} X_{i A}\left(\varphi_{g, A}^{i}(v)\right) .
$$

Since $\varphi_{g, A}^{i}$ are homogeneous, $\left[g, X_{A}(v)\right]=1$ for $v \in V_{A}$ implies $\left[g, X_{A}(\lambda v)\right]=1$ for any $\lambda \in R$. Also by [5, Th. 2], there exist a set of homogeneous polynomial maps $q_{A}^{i}: V_{A} \times V_{A} \rightarrow V_{i A}$, $i>1$, such that

$$
X_{A}(v) X_{A}(w)=X_{A}(v+w) \prod_{i>1} X_{i A}\left(q_{A}^{i}(v, w)\right)
$$

for all $v, w \in V_{A}$. Assume that $\left[g, X_{A}(v)\right]=\left[g, X_{A}(w)\right]=1$. Then

$$
g X_{A}(v+w) g^{-1}=g X_{A}(v) X_{A}(w) g^{-1} \cdot g\left(\prod_{i>1} X_{i A}\left(q_{A}^{i}(v, w)\right)\right)^{-1} g^{-1}=1,
$$

since by inductive hypothesis $g$ centralizes $X_{i A}\left(V_{i A}\right)$ for all $i>0$.

\section{The proof}

Proof of Theorem 1. Let $g \in G(R)$ centralize $E(R)=E_{Q}(R)$, where $Q$ a strictly proper parabolic subgroup of $G$. We are going to show that $g \in \operatorname{Cent}(G)(R)$. By Lemma 1 it is enough to show that $g \in \operatorname{Cent}(G)\left(R_{M}\right)$ for any maximal ideal $M$ of $R$. Fix an ideal $M$, and set $R^{\prime}=R_{M}$. Let $P$ be a minimal parabolic subgroup of $G_{R^{\prime}}$. By Lemma 2 for any $A \in \Phi_{P}$ there is a system of generators $e_{A i}, 1 \leq i \leq n_{A}$, of the $R^{\prime}$-module $V_{A}$ such that one has $\left[g, X_{A}\left(e_{A i}\right)\right]=1,1 \leq i \leq n_{A}$. Note that $\Phi_{P}$ is a root system by [2, Exp. XXVI, §7], and by the assumption of the theorem all irreducible components of $\Phi_{P}$ are of rank $\geq 2$.

Let $\amalg$ Spec $S_{\tau} \rightarrow$ Spec $R^{\prime}$ be an fpqc-covering such that $G$ splits over each Spec $S_{\tau}$. It is enough to check that $g \in \operatorname{Cent}(G)\left(S_{\tau}\right)$ for every $\tau$ (here we identify $g$ with its image under $\left.G\left(R^{\prime}\right) \rightarrow G\left(S_{\tau}\right)\right)$. Fix one $\tau$, and set $S=S_{\tau}$ for short. Again by Lemma 1 it is enough to show that $g \in \operatorname{Cent}(G)\left(S_{N}\right)$ for any maximal ideal $N$ of $S$.

Since a system of generators $e_{A i}, 1 \leq i \leq n_{A}$, of the $R^{\prime}$-module $V_{A}$, also generates $\left(V_{A} \otimes_{R^{\prime}} S\right) \otimes_{S} S_{N}$ as an $S_{N}$-module, $g$ satisfies the conditions of Lemmas 3 and 6 (for the base 
ring $\left.S_{N}\right)$; hence $g \in L\left(S_{N}\right)$, where $L$ is a Levi subgroup of $P$. By Lemma 7 this implies that $g$ centralizes $E\left(S_{N}\right)$. Since $G_{S_{N}}$ is split, it has a Borel subgroup $B$, and $E\left(S_{N}\right)=E_{B}\left(S_{N}\right)$. Applying Lemmas 3 and 6 to $B$ instead of $P$, we get that $g \in T\left(S_{N}\right)$ for a split maximal subtorus $T$ of $G_{S_{N}}$. Hence $g \in \operatorname{Hom}\left(\Lambda / \Lambda_{r}, S_{N}\right) \subseteq \operatorname{Hom}\left(\Lambda, S_{N}\right)=T\left(S_{N}\right)$, where $\Lambda$ is the weight lattice of $G$, and $\Lambda_{r}$ is the root sublattice. Therefore, $g \in \operatorname{Cent}(G)\left(S_{N}\right)$.

\section{References}

[1] E. Abe, J. F. Hurley, Centers of Chevalley groups over commutative rings, Comm. in Algebra 16 (1988), 57-74.

[2] M. Demazure, A. Grothendieck, Schémas en groupes, Lecture Notes in Mathematics, Vol. 151-153, Springer-Verlag, Berlin-Heidelberg-New York, 1970.

[3] A. Luzgarev, A.Stavrova, Elementary subgroup of an isotropic reductive group is perfect, http://arxiv.org/abs/1001.1105; to appear in St. Petersburg Mathematical Journal.

[4] H. Matsumoto, Sur les sous-groupes arithmétiques des groupes semi-simples déployés, Ann. Sci. de l'É.N.S. $4^{e}$ série, tome 2, n. 1 (1969), 1-62.

[5] V. Petrov, A. Stavrova, Elementary subgroups of isotropic reductive groups, St. Petersburg Math. J. 20 (2009), 625-644.

[6] V. Petrov, A. Stavrova, Tits indices over semilocal rings, to appear in Transformation Groups.

[7] A. Stavrova, Normal structure of maximal parabolic subgroups in Chevalley groups over rings, Algebra Colloq. 16 (2009), 631-648.

[8] W. C. Waterhouse, Introduction to affine group schemes, Springer-Verlag, New York, 1979. 